www.jmscr.igmpublication.org

Impact Factor 5.84

Index Copernicus Value: 71.58

ISSN (e)-2347-176x ISSN (p) 2455-0450

crossref DOI:_https://dx.doi.org/10.18535/jmscr/v5i11.139

Journal Of Medical Science And Clinical Research

\title{
The Effect of Synbiotics on the Duration of Crying Time in Infants with Abdominal Colic
}

Authors

\author{
T.Vijayalakshmi ${ }^{1}$, C.S.Balachandran ${ }^{2}$, S.Chidambaranathan $^{3}$
}

${ }^{* 1}$ Post Graduate, Department of Paediatrics, RMMCH, Chidambaram-608002, Tamilnadu, India

${ }^{2}$ Professor, Department of Paediatrics, RMMCH, Chidambaram-608002, Tamilnadu, India

${ }^{3}$ Associate Professor, Department of Paediatrics, RMMCH, Chidambaram-608002, Tamilnadu, India

\begin{abstract}
Objectives: Infantile colic is a condition which is seen commonly in infants that has psychosocial effects. The objective of this study was to evaluate the effectiveness of synbiotics with 4 bacterial strains Streptococcus faecalis, Clostridium butyricum, Bacillus mesentricus and Lactobacillus sporogenes as an add on therapy to standard treatment for the management of infantile colic by measuring the reduction in mean daily crying time.

Materials and Methods: An observational, randomized, clinical study was done in Dept of Pediatrics, RMMCH, Annamalai University, Chidambaram involving fifty breastfed infants aged 30 - 180 days with abdominal colic. They were alloted into two groups- Group A and Group B by randomization. Infants in Group A were given standard treatment with Dimethicone for 5 days and infants in Group B were given standard treatment for 5 days and synbiotic for 28 days. Parents had been asked to record the crying times and the infants were followed up and the reduction in mean crying time (100\% reduction) was noted on day 7,14 and 28.
\end{abstract}

Results: Infants assigned to synbiotic group showed rapid improvement in terms of reduction in mean daily crying time (100\% reduction - P 0.001) in comparison to standard treatment alone.

Conclusion: It was concluded from this study that it is safe, effective and beneficial to use synbiotic as an add on therapy to standard treatment to manage abdominal colic in infants.

Keywords: Infantile colic, synbiotic, Reduction in crying time.

\section{Introduction}

Infantile colic refers to a clinical condition with inconsolable crying, fussing and irritability in a healthy infant during the first 6 months of life. ${ }^{(1)}$ Although the incidence is 5\% -19\% among younger infants, it remains a frustrating problem for the parents and can bring about significant psychosocial outcomes. $^{(2)}$

Infantile colic is described clinically as Wessel's criteria of crying and/or fussing $\geq 3$ hours/day for $\geq$ three days/week for $\geq$ three weeks. Later there came the modified Wessel's criteria of crying and/or fussing $\geq$ three hours/day for $\geq 3$ days/ week for one week which was used for practical purpose and it starts around 3 weeks, peaking at 8 weeks, and remitting past 12 weeks of age. ${ }^{(3)}$

Infant crying has been associated to psycological problems where the infant is hypersensitive and irritable. $^{(4)}$ 
Although many researches were done on the etiology of infantile colic for the past 40 years, it still remains unclear. There are many causes for colicky pain including behavioural, food hypersensitive reaction and allergy, immature nature of the gut and its function and abnormal motility of the intestine. ${ }^{(2)}$ It can arise because of the disruption or immaturity of immune and epithelial barrier leading to the inflammation of the colon. ${ }^{(5)}$

Some studies have confirmed the abnormal levels of fecal calpotectin, which is seen in infection and inflammation of colon in infants with abdominal colic. $^{(6)}$

Recent studies have proved that intestinal microbiota plays a major part in the pathophysiology of infantile colic and that dysbiosis (altered gut microbiota) affects the motility of the intestine and production of gas in infantile colic. ${ }^{(7)}$

It is also concluded from the earlier studies that manipulation of microbiota can result in symptomatic improvement and one such approach is using probiotics or prebiotics. Probiotic is an oral dietary supplements which contains a enough quantity of viable microorganisms having the beneficial effects of altering the intestinal flora of the host. Prebiotics is a nondigestible substances that augments the activity of one or more indigenous probiotic bacteria. Synbiotic is a substance that has both probiotics and prebiotics in it. ${ }^{(8)}$

The probiotics have a direct metabolic effect by providing enzymatic activities in the intestinal lumen and also interacts with the mucosal and epithelial layer of the intestine enhancing the barrier effects, digestive processes, mucosal immune system and enteric nervous system. ${ }^{(9)}$

\section{Materials and Methods}

An observational, randomized, clinical study was done in the Dept of Pediatrics, Rajah Muthiah Medical college and hospital (RMMCH), Annamalai University, Chidambaram, Tamilnadu involving infants with abdominal colic from March 2016 after getting clearance from the
Institutional Ethics Committee, RMMCH, Annamalai university and conducted as per the International conference on Harmonization-Good clinical Practice (ICH-GCP) pointers.

Infants of both sexes, aged 30 to 180 days, exclusively breastfed with infantile colic described as per modified wessel's criteria were included in the study after obtaining consent from their parents and after explaining about the purpose of the study.. Infants with chronic infection, any organic cause for abdominal pain and any history of prior antibiotic or synbiotic supplementation at some stage in 2 weeks earlier had been excluded.

All infants were alloted into 2 groups by randomization- Group A and Group B.Infants in Group A were given standard treatment with Dimethicone for 5 days and infants in Group B were given standard treatment for 5 days and synbiotic for 28 days. Each $5 \mathrm{ml}$ of oral synbiotic contained

- Streptococcus faecalis T-one hundred ten 30 million

- Clostridium butyricum TO-A - 2 million

- Bacillus mesentricus TO-A - 1 million

- Lactobacillus sporogenes- 50 million

Parents had been asked to document the period of crying time and any adverse events every day for 28 days. The infants were followed up and the reduction in mean crying time (100\% reduction) was noted on day 7,14 and 28 .

The statistical analysis was performed. We used the student $t$ test for comparing mean values of continuous variables approximating normal distribution. For non normally distributed variables, the Mann-Whitney U test was used. The difference between study groups was considered significant when the $\mathrm{p}$ value was $<0.05$. All statistical tests were two tailed and performed at the $5 \%$ level of significance.

\section{Results}

Of the 64 colicky infants attended OPD, 50 colicky infants who fulfilled the inclusion criteria were enrolled. Among them, 25 infants were 
treated with standard treatment alone (Group A) and 25 infants with Synbiotic along with standard treatment (Group B). No infants were lost follow up and there was no adverse effect seen in the present study.

The mean daily crying time on day 0 for Group A (standard treatment alone) was $129.60 \pm 41.280$ (range: 60 - $180 \mathrm{~min} / \mathrm{episode)}$ and for Group B (synbiotic and standard treatment) was $117.60 \pm$ 47.371 (range : $60-180 \mathrm{~min} /$ episode). The mean daily crying time for Group A and Group B on subsequent visits i.e on day 7,14 and 28 were shown in Table 1.

The reduction in mean daily crying time on day 7 , 14 and 28 between group A and Group B was shown in Fig 1.
On Day 7 , the reduction in mean crying time in Group B $-100 \%$ reduction (Excellent) was seen among $48 \%$ of infants which was statistically significant (P- 0.001) in comparison to Group A where $100 \%$ reduction was not seen in any of the infants. On day 14 , the reduction in mean crying time in Group B - $100 \%$ reduction (Excellent) was seen among $84 \%$ of infants which was statistically significant (P- 0.046) when compared to Group A where $100 \%$ reduction was seen among $52 \%$ of infants. On day 28 , the reduction in mean crying time in Group B - $100 \%$ reduction (Excellent) was seen among $100 \%$ of infants which was statistically significant (P0.037) in comparison to $84 \%$ of infants in Group A. The outcome of crying time in standard and synbiotic group was shown in table 2 .

Table 1. Mean daily crying time on day 0 and subsequent visits

\begin{tabular}{|l|c|c|c|}
\hline Day & \multicolumn{3}{|c|}{ Mean Daily crying time (mins) } \\
\hline & Group A $(\mathrm{n}=25)$ & Group B $(\mathrm{n}=25)$ & P value \\
\hline 0 & $129.60 \pm 41.280$ & $117.60 \pm 47.371$ & 0.352 \\
\hline 7 & $72.00 \pm 35.707$ & $33.60 \pm 40.915$ & 0.001 \\
\hline 14 & $25.20 \pm 32.31$ & $4.80 \pm 11.225$ & 0.007 \\
\hline 28 & $4.80 \pm 11.225$ & $0.00 \pm 0.00$ & 0.039 \\
\hline
\end{tabular}

Fig 1 Reduction in mean daily crying time

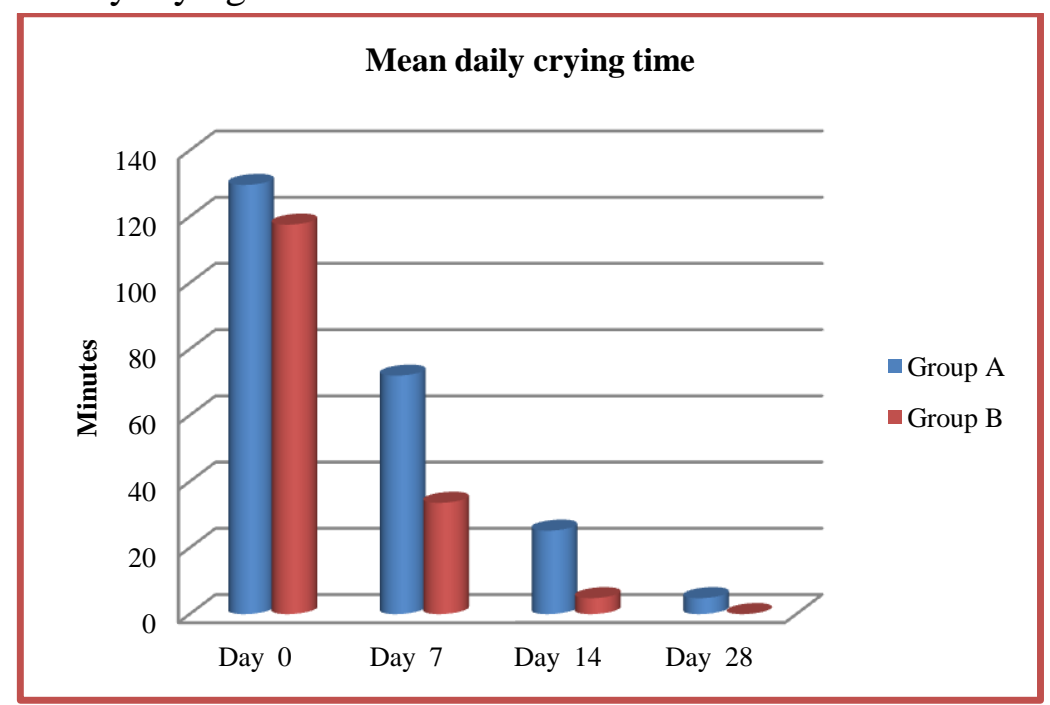

Table 2 Outcome of crying time in standard and synbiotic group

\begin{tabular}{|c|c|c|c|}
\hline \multirow{2}{*}{$\begin{array}{l}\text { Reduction in mean } \\
\text { daily crying time }\end{array}$} & \multicolumn{2}{|c|}{$\begin{array}{cc}\text { Inference } \\
(100 \% \text { reduction-excellent })\end{array}$} & \multirow{2}{*}{$P$ value } \\
\hline & $\begin{array}{c}\text { Group A-standard treatment } \\
\text { alone n }(\%)\end{array}$ & $\begin{array}{l}\text { Group B-synbiotic } \\
\text { group n (\%) }\end{array}$ & \\
\hline Day 7 & $0(0 \%)$ & $12(48 \%)$ & 0.001 \\
\hline Day 14 & $13(52 \%)$ & $21(84 \%)$ & 0.046 \\
\hline Day 28 & $21(84 \%)$ & $25(100 \%)$ & 0.037 \\
\hline
\end{tabular}




\section{Discussion}

In the present study, we used a synbiotic containing four bacterial strains. The reduction in crying time was significantly higher in synbiotic group (Group B) when compared to standard treatment alone (Group A). The results were impressive as $48 \%$ of infants in synbiotic group showed $100 \%$ reduction in crying time at day 7 compared with $0 \%$ of infants in Group A. No adverse events were seen in the present study.

Savino et al. In 2007 showed the effectiveness of lactobacillus reuteri strain ATCC 55730 in comparison with simethicone in the treatment of infantile colic by recording the reduction of more than $50 \%$ of crying time on day $28 .^{(10)}$

The previous study done by savino et al. in 2007 was duplicated in 2010 but with different strain, lactobacillus reuteri DSM17938 in comparison with the placebo where the crying time $(>50 \%$ reduction) was recorded on day 7 (8 vs. 20 , respectively; $\mathrm{P}=0.006)$, day 14 (13 vs. 24 , respectively; $\mathrm{P}=0.007)$, and day 21 (15 vs. 24 , respectively; $\mathrm{P}=0.036){ }^{(11)}$

Szajewska et al. In 2013 used the same probiotic strain-lactobacillus reuteri DSM17938 and reduction in crying time $(>50 \%)$ were recorded on day 7,21 and $28 .^{(12)}$

Kianifer et al. in 2014 in his study enrolled 50 breastfed infants in the age group of 15-120 days with infantile colic and used synbiotic containing L casei, L rhamnosus, Streptococcus thermophilus, Bifidobacterium breve, L acidophilus, B infantis, L bulgaricus, and fructooligosaccharides. He compared with the placebo and found out that there was a reduction of $>50 \%$ in crying time among $87 \%$ of infants in synbiotic group and 46 $\%$ of infants in placebo group at 30 days $(\mathrm{P}<0.01){ }^{(13)}$

\section{Importance of type and number of probiotic strains}

The beneficial effects of synbiotics depends on the type of strains, number of strains and duration of intervention. kianifer et al in 2014 in his study used a probiotic strain with $109 \mathrm{CFU}$ combined with prebiotics - fructooligosaccharides. ${ }^{(13)}$
Compared to this study, in the present study we used a synbiotic with a combination of dose of lactobacillus sporogenes $5 \times 10^{7}$, Streptococcus faecalis $3 \times 10^{7}$, Clostridium butyricum $2 \times 10^{6}$ and Bacillus mesentericus $1 \times 10^{6} \mathrm{CFU}$.

\section{Conclusion}

It can be concluded from this study that the use of synbiotic was effective in reducing the crying time and can be used as an add on therapy to standard treatment in managing infantile colic.

\section{References}

1. Savino F, Tarasco V: New treatments for infant colic. Current Opinion in Pediatrics 2010, 22:791-7.

2. Anabrees, J., Indrio, F., Paes, B. \& Alfaleh K. Probiotics for infantile colic: a systematic review. BMC Pediatr. 2013, 13: 186.

3. Valerie Sung, Harriet Hiscock, Mimi Tang, Fiona K Mensah, Ralf G Heine, Amanda Stock, Elissa York, Ronald G Barr and Melissa Wake Probiotics to improve outcomes of colic in the community: Protocol for the Baby Biotics randomised controlled trial, BMC Pediatrics;2012-12:135

4. Sumitha Nayak, Infantile Colic -Do we understand the Etiology?, march 2011,vol 2;issue 1

5. Eberi G,Lochner M. The development of intestinal lymphoid tissues at the interface of self and microbiota. Mucosal Immunol.2009, 155:823-8

6. Rhoads JM, Fatheree NY, Norori J, Liu Y, Lucke JF, Tyson JE, et al. Altered fecal microflora and increased fecal calprotectin in infants with colic. J Pediatr2009; 155:823-8.

7. de Weerth C, Fuentes S, Puylaert P, et al. Intestinal microbiota of infants with colic: development and specific signatures. Pediatrics 2013; 131: $550-558$.

8. Dan W. Thomas, MD, Frank R. Greer, $\mathrm{MD}$, and the Committee on Nutrition and 
the Section on Gastroenterology,

Hepatology, and Nutrition., Probiotics and Prebiotics in Pediatrics, 2010,126: 1217

9. Ger T. Rijkers, Stig Bengmark, Paul Enck, Dirk Haller, Udo Herz, Marko Kalliomaki, Satoshi Kudo et al. Guidance for Substantiating the Evidence for Beneficial Effects of Probiotics: J. Nutr.2010, 140: 671S-676S.

10. Savino F,Pelle E, Palumeri E,Oggero R,Miniero R.Lactobacillus reuteri ATCC 55730 vs simethicone in the treatment of infantile colic: a prospective randomized study. Pediatrics 2007; 119;e124-30

11. Savino F, Cordisco L, Tarasco V, et al. Lactobacillus reuteri DSM 17938 in infantile colic: a randomized, double-blind, placebo-controlled trial. Pediatrics 2010;126: 526-33.

12. Szajewska, H., Gyrczuk, E. \& Horvath, A. (2013) Lactobacillus reuteri DSM 17938 for the management of infantile colic in breastfed infants: A randomized, doubleblind, placebo-controlled trial. J. Pediatr., 162, 257-262.

13. Kianifar H, Ahanchian H, Grover Z, et al. Synbiotic in the management of infantile colic: a randomised controlled trial. $\mathrm{J}$ Paediatr Child Health 2014; 50:801-5. 